Berting, A.; Schultheiß, Birgit; Obstoj, Peter; Henning, Günter:

Diagnostik arterieller Gefäßerkrankungen mittels Klassifikation peripherer und kardialer Impedanzsignale

\footnotetext{
Zuerst erschienen in: $\quad$ Biomedizinische Technik = Biomedical Engineering. - Berlin [u.a.] : de Gruyter. - 47 (2002), S1b, S. 812-815.

Jahrestagung der Deutschen Gesellschaft für Biomedizinische Technik (DGBM) im VDE ; 36 (Karlsruhe) : 2002.09.25-27

Erstveröffentlichung: 2002

Datum Digitalisierung: $2009-11-26$

ISSN (online): $\quad$ 1862-278X

ISSN(print) 0013-5585

DOI: $\quad$ 10.1515/bmte.2002.47.s1b.812

[Zuletzt gesehen: 2019-12-12]
}

„Im Rahmen der hochschulweiten Open-Access-Strategie für die Zweitveröffentlichung identifiziert durch die Universitätsbibliothek IImenau."

"Within the academic Open Access Strategy identified for deposition by IImenau University Library."

„Dieser Beitrag ist mit Zustimmung des Rechteinhabers aufgrund einer (DFGgeförderten) Allianz- bzw. Nationallizenz frei zugänglich."

„This publication is with permission of the rights owner freely accessible due to an Alliance licence and a national licence (funded by the DFG, German

Research Foundation) respectively."

\section{DFG}

Nationallizenzen 


\title{
DIAGNOSTIK ARTERIELLER GEFÄßERKRANKUNGEN MITTELS KLAS- SIFIKATION PERIPHERER UND KARDIALER IMPEDANZSIGNALE
}

\author{
A. Berting, B. Schultheiß, P. Obstoj, G. Henning \\ Institut für Biomedizinische Technik und Informatik, Technische Universität Ilmenau, Deutschland \\ andreas.berting@stud.tu-ilmenau.de
}

\begin{abstract}
Verschiedene Zeitbereichsparameter sowie aus der Wavelet-Transformation gewonnene Merkmale wurden hinsichtlich ihrer Eignung zur Frühdiagnostik peripherer Gefäßerkrankungen untersucht. Der Untersuchung liegen periphere und zentrale Impedanzsignale von 78 Probanden und 74 Dialysepatienten, die im Rahmen ihrer Therapie auf entsprechende Gefäßerkrankungen untersucht wurden, zugrunde. Zur diagnostischen Entscheidungsfindung wird ein Klassifikationsbaum verwendet. Die kombinierte Betrachtung zentraler und peripherer Kurven gestattet in Verbindung mit den Wavelet-Koeffizienten eine differenziertere Beurteilung des arteriellen Gefäßsystems als bisher bekannte, auf der Analyse von Kurven nur eines Messabschnittes beruhende Methoden.
\end{abstract}

Keywords Gefäßerkrankung, Bioimpedanz, WaveletTransformation, Klassifikationsbaum

\section{Einleitung}

In der Bundesrepublik Deutschland leiden über 3 Mio. Menschen an einer peripheren arteriellen Verschlusskrankheit (paVk) [1]. Die Folgen der dadurch bedingten Minderdurchblutung reichen von Schmerzen beim Gehen und Einschränkungen der Beweglichkeit bis hin zu Amputationen und lebensbedrohlichen Komplikationen, wie z.B. Sepsis. Strukturelle Gefäßveränderungen, welche die Entstehung einer paVk verursachen, sind oft auch im Bereich thorakaler Gefäße zu finden, so dass bei Patienten mit paVk häufig auch sklerotische Veränderungen großer Arterien, eine koronare Herzkrankheit sowie zerebrovaskuläre Erkrankungen beobachtet werden [1-3]. Die frühzeitige Erkennung und Therapie von arteriellen Gefäßerkrankungen ist somit geboten.

Zur Diagnostik peripherer arterieller Verschlusskrankheiten werden in der klinischen Praxis neben der peripheren Dopplerdruckmessung auch plethysmographische Verfahren, wie z.B. die Impedanz-Plethysmographie, eingesetzt [4]. Die diagnostische Klassifikation beruht hierbei in der Regel auf einzelnen Amplituden- bzw. Formparametern der peripheren Pulswelle, wobei in der klinischen Routine Informationen über die tatsächliche kardiale Auswurfleistung nur teilweise verfügbar sind bzw. diese oft nicht in die Befundung einbezogen werden.

Im Rahmen dieses Beitrages soll durch eine Bewertung kardialer Impedanzparameter sowic durch Einbeziehung zeilaufgelöster Frequenzinformationen (Wavelet-Transformation) eine Erweiterung des zur Diagnostik verwendeten Parametersatzes erfolgen und damit dic diagnostische Zuverlässigkeit des Verfahırens erhölıt werden.

\section{Materialien und Methoden}

Basierend auf peripheren und thorakalen Impedanzsignalen von 78 Probanden und 74 Dialysepatienten, die im Rahmen ihrer Therapie auf entsprechende Gefäßerkrankungen untersucht wurden, sind Zeitbereichsparameter sowie aus der Wavelet-Transformation gewonnene Merkmale auf ihre Eignung zur Frühdiagnostik peripherer $\mathrm{Ge}$ fäßerkrankungen untersucht worden. Die ImpedanzPlethysmographie erfolgte mit dem Monitoring-System multiscreen (medis GmbH, Deutschland), wobei zur Signalerfassung die in Abbildung 1 dargestellte Elektrodenanordnung angewendet wurde. Dabei dienen die am Thorax applizierten Elektroden zur Akquisition von EKG und zentralem Impedanzsignal, während über die am Bein im Bereich der Arteria tibialis angeordneten Elektroden die periphere Pulswelle (PPW) erfasst wird. Die Signale wurden mit einer Abtastfrequenz von $200 \mathrm{~Hz}$ aufgezeichnet.

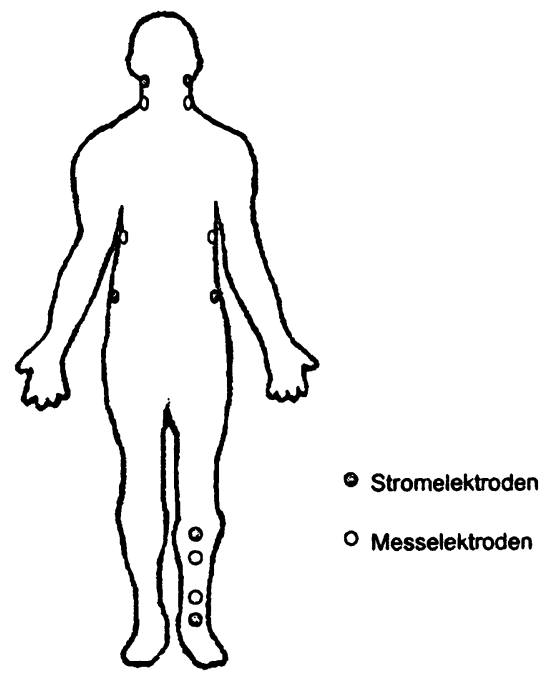

Abbildung 1: Elektrodenkonfiguration

Abbildung 2 zeigt anhand der PPW eines ,gefäßgesunden“ Probanden die Definition der Parameter Gipfelamplitude (GA) und Gipfelbreite (GB). Neben den in Abbildung ? dargestellten Parametern erfolgt eine Analyse der mavimalen Steigung (MA) des anakroten Schenkels der PPII, der Propagationszeit (PT) [4], der Zeitspanne (TA) zMischen der Q-Zacke im E.KG und dem Maximum der ensten Ableitung von PPW, des Verhältnisses von (iA zur (icwebegrundimpedanz (Z0) -. so genannter Impedaulquoticnt (IQ) - und des als Ansticgsquotiont (AQ) bercichnctin Verhältuisses von MA zu 70).

Darüber hinaus werden basicrond auf der ersien Dotallin. formation (DI) nach ciner Warclet-Transfornatton dor 
PPW und deren erster Ableitung nach der Zeit Bildbereichsparameler (Leislungskennzahlen von DI in verschicdenen Kurvenabschnilten) definiert.

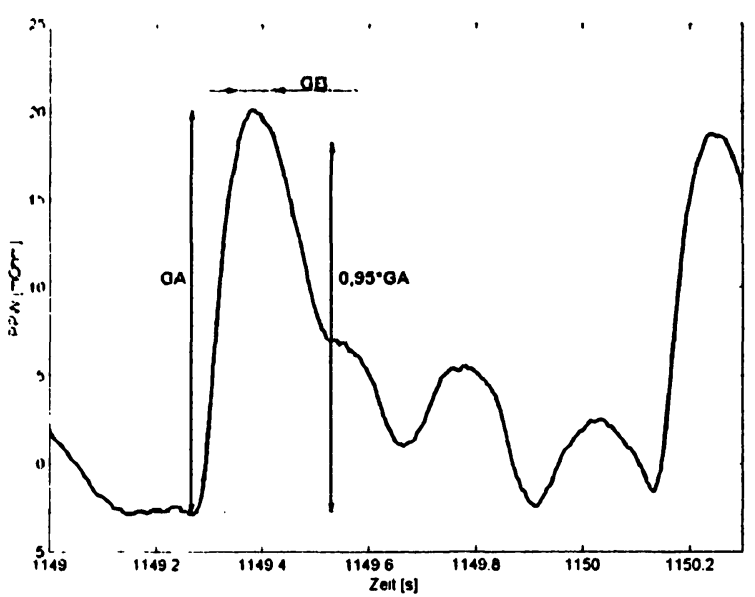

Abbildung 2: Parameter der peripheren Pulswelle

Zur Einschätzung der kardialen Leistung werden im zentralen Impedanzsignal den peripheren Parametern (GA, GB und $\mathrm{MA}$ ) analoge Merkmale bestimmt und analysiert. Weiterhin werden wesentliche hämodynamische Parameter, wie z.B. die Ejektionsfraktion (EF), deren Bestimmung aus dem Impedanzkardiogramm (IKG) erfolgt, betrachtet. Eine Bestimmung der Bildbereichsparameter erfolgt in Analogie zur peripheren Analyse.

Mittels Varianzanalyse $(p<0,01)$ werden die aus den peripheren und zentralen Signalen extrahierten Parameter auf ihre diagnostische Signifikanz getestet. Zur weiteren Verbesserung der Separationsfähigkeit der erhaltenen Merkmale werden Parametertransformationen (Differenzen- und Quotientenbildungen) durchgeführt.

Die berechneten Parameter aus Zeit- und Bildbereich dienen der Klassifikation in die Diagnosegruppen ,gefäßgesund" (Gruppe „ok“), ,periphere arterielle Verschlusskrankheit“ (Gruppe „paVk“), „verminderte periphere Durchblutung infolge kardialer Erkrankungen" (Gruppe ,zentral“) bzw. ,allgemein gefäßkrank“ (Gruppe „allgemein"). Unter Anwendung des Programmpaketes STATISTICA erfolgt die Zuordnung in die beschriebenen Diagnosegruppen durch Klassifikationsbäume mit anschließender Bewertung der Ergebnisse durch Kreuzvalidierung. Die Lernstichprobe setzt sich aus 78 Probanden (51 männlich, 27 weiblich, Durchschnittsalter 35,9 $\pm 13,7$ Jahre), die a priori als ,gefäßgesund“ angenommen werden, und aus 23 ,gefäßgesunden" Dialysepatienten zusammen. 21 Patienten leiden exklusiv an zentralen Gefäßresp. Herzerkrankungen, 12 Patienten exklusiv an peripherer arterieller Verschlusskrankheit und bei 18 Patienten wurden sowohl periphere als auch zentrale Gefäßprobleme diagnostiziert. Die in der untersuchten Patientengruppe (32 männlich, 42 weiblich, Durchschnittsalter $66,2 \pm 13,5$ Jahre) auftretenden Erkrankungen, welche die Impedanzsignale beeinflussen können, fasst Tabelle 1 zusammen.
Tabelle I: Relevante Begleiterkrankungen der untersuchten Patientengruppe

\begin{tabular}{lc}
\hline Erkrankung & AnzahI \\
\hline Ilypertonic (WHO II/III) & $38(51,3 \%)$ \\
Herzinsuffizienz (NYHA II-IV) & $9(12,1 \%)$ \\
koronare Herzkrankheit & $31(41,9 \%)$ \\
linksventrikuläre Hypertrophie & $14(18,9 \%)$ \\
Diabctes mellitus & $33(44,6 \%)$ \\
periphere arterielle Verschlusskrankheit & $30(40,5 \%)$ \\
(Stadium II-IV nach Fontaine) & $30(40,5 \%)$ \\
zentrale Gefäß- und Klappenprobleme & 30
\end{tabular}

\section{Ergebnisse}

Abbildung 3 zeigt die bei paVk auftretenden Amplitudenund Formveränderungen der peripheren Pulswelle. Bei "gefäßgesunden“ Probanden ist die PPW durch einen steilen Anstieg, einen schmalen, runden Gipfel und dikrote Nachwellen gekennzeichnet. Im Gegensatz dazu zeigen die peripheren Impedanzsignale bei Patienten mit paVk erniedrigte Gipfelamplituden, verbreiterte Gipfel und aufgrund der geringeren Arterienwandelastizität verschwindet die Dikrotie weitgehend. Die Analyse der ersten zeitlichen Ableitungen der peripheren Pulswellen aus Abbildung 3 bestätigt die Vermutung, dass der anakrote Schenkel des peripheren Impedanzsignals bei Vorliegen einer paVk eine geringere maximale Steigung aufweist.
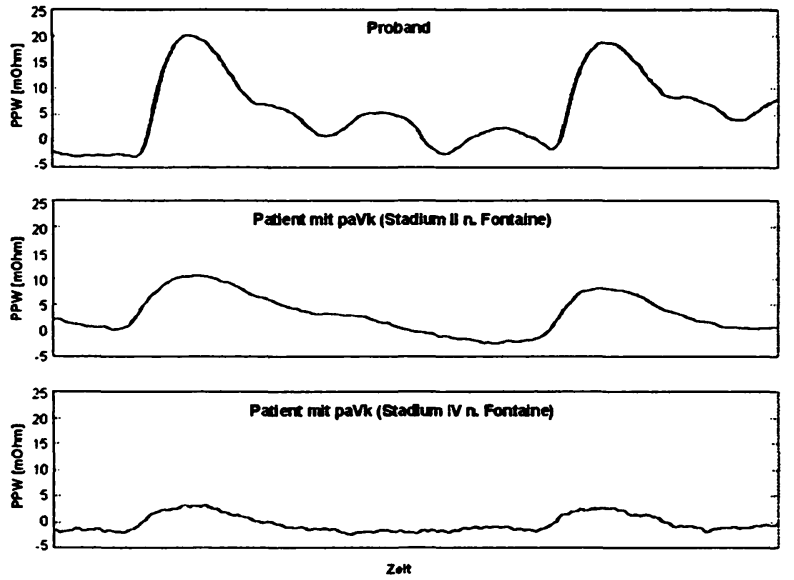

Abbildung 3: Vergleich charakteristischer PPW-Formen von Probanden und Patienten mit paVk verschiedenen Grades

Im Ergebnis einer Varianzanalyse zeigen IQ und AQ signifikante Mittelwertdifferenzen $(p<0,01)$. Abbildung 4 verdeutlicht jedoch, dass eine alleinige Verwendung der $\mathrm{Pa}-$ rameter AQ bzw. IQ keine zufrieden stellenden Klassifikationsergebnisse liefern würde.

Die anderen Zeitparameter zeigen mitunter ebenfalls signifikante Mittelwertdifferenzen, weisen aber größere Überschneidungsbereiche der Diagnosegruppen im BoxWhisker-Plot auf. 

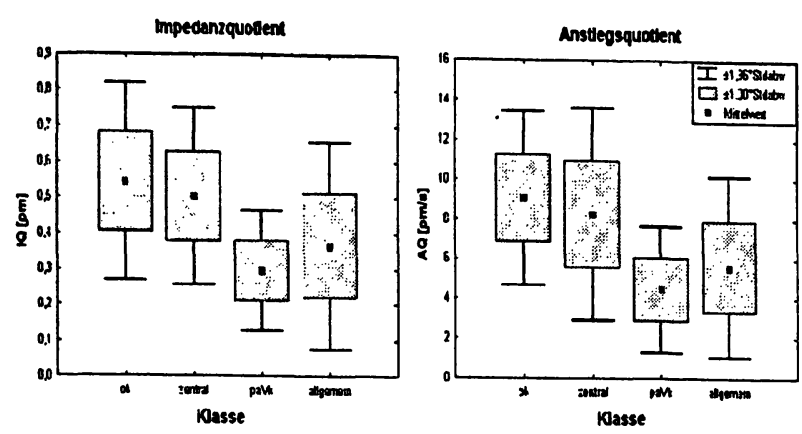

Abbildung 4: Box-Whisker-Plot der Parameter IQ und AQ, $(\mathrm{pm}=$ pro mille)

Entgegen der Erwartung, dass zentrale Gefäß- und Herzprobleme die periphere Pulskurve in ihrer Form maßgeblich beeinflussen, muss bei Betrachtung von Abbildung 4 festgestellt werden, dass im Vergleich der Gesunden (Gruppe „ok") mit den zentral geschädigten Patienten (Gruppe ,zentral") nur geringe, nicht signifikante Abweichungen der Gruppenmittelwerte von AQ und IQ auftreten. Die zusätzliche Betrachtung der vom Messsystem multiscreen bereitgestellten Zeitbereichsparameter des zentralen Impedanzsignals sowie des Impedanzkardiogrammes zeigt vor allem deutliche Veränderungen in der Gruppe der kardial erkrankten Patienten, lässt aber keine deutliche Verbesserung hinsichtlich der Diagnostik peripherer arterieller Verschlusskrankheiten erwarten.

Abbildung 5 zeigt die periphere Pulswelle eines „gefäßgesunden" Probanden mit der zugehörigen ersten Detailinformation (D1) nach einer Wavelet-Transformation. Als Parameter werden die mittleren Leistungen von D1 im anakroten Teil zwischen beg (Beginn systolischer Anstieg und max (Zeitpunkt des Maximums von PPW), im katakroten Teil zwischen max und end (empirisch bestimmte Detektionsgrenze) sowie die mittlere Gesamtleistung von D1 extrahiert. Analoge Parameter werden für die erste Detailinformation der zeitlichen Ableitung von PPW bestimmt.
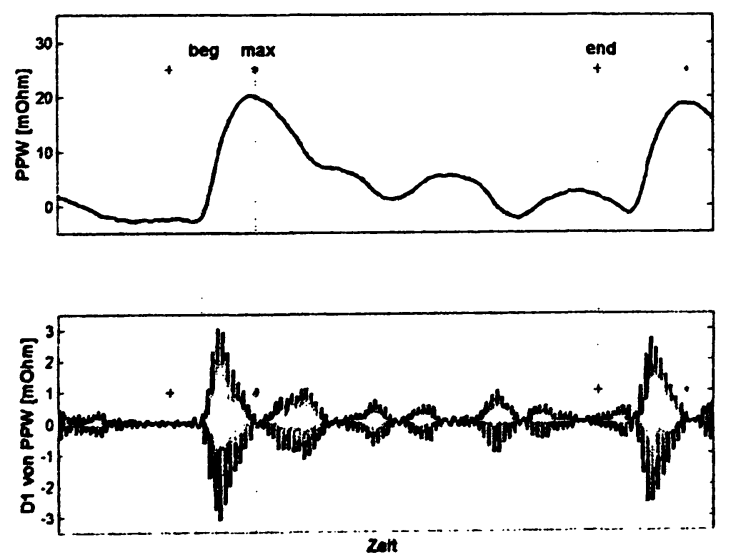

Abbildung 5: repräsentative periphere Pulswelle (oben) und erste Detailinformation von PPW (unten) eines Probanden

Die veränderten Frequenzinformationen, besonders im katakroten Schenkel der PPW bei ciner paVk, verdeutlicht Abbildung 6. Wie sich im Ergebnis unserer Untersuchun- gen zeigt, liefern auch Frequenzinformationen des anakroten Teils einen Beitrag zur Diagnostik (Klassifikation).
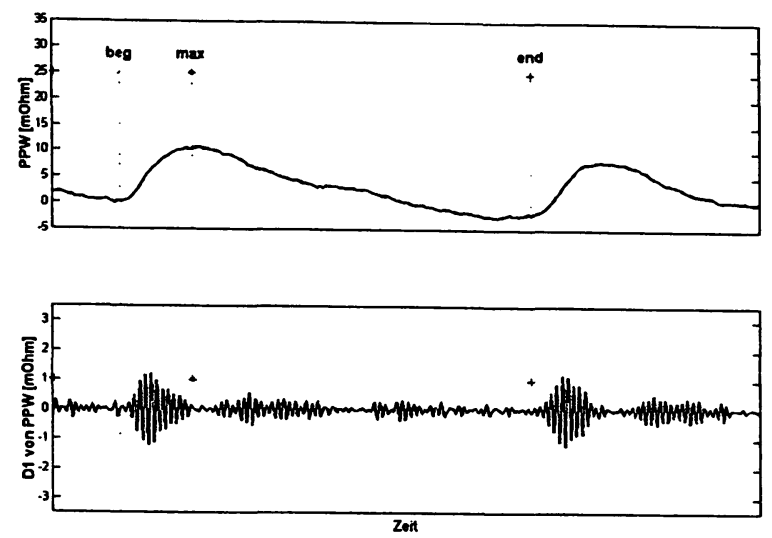

Abbildung 6: periphere Pulswelle (oben) und erste Detailinformation von PPW (unten) eines Patienten mit paVk (Stadium II nach Fontaine)

In einer Reproduzierbarkeitsanalyse wurde festgestellt, dass die intraindividuellen Schwankungen sämtlicher $\mathrm{Pa}$ rameter geringer sind als die interindividuellen Abweichungen. Eine Abhängigkeit der Klassifikationsergebnisse vom Messzeitpunkt während einer Dialysebehandlung konnte nicht festgestellt werden.

Im Vergleich zur alleinigen Analyse von Zeitbereichsparametern führt die Verwendung eines Klassifikationsbaumes (s. Abbildung 7) basierend auf Zeit- und Bildbereichsparametern der peripheren Signale zu einer Verbesserung des Ergebnisses nach 10-facher Kreuzvalidierung.

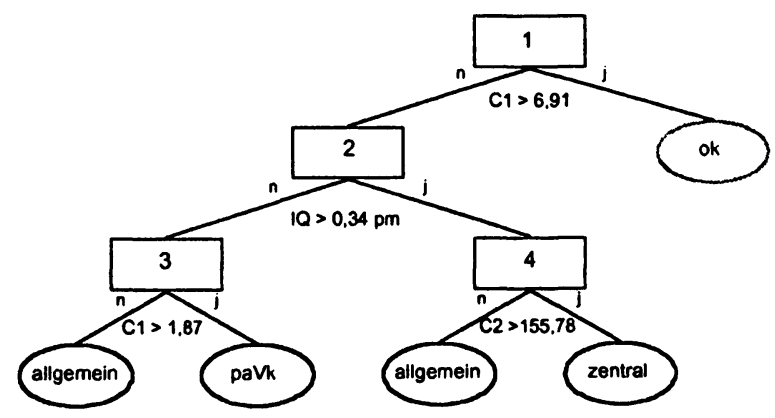

Abbildung 7: Klassifikationsbaum zur Einteilung in vier Diagnosegruppen

Die Struktur des in Abbildung 7 dargestellten Klassifikationsbaumes wurde anhand der erzielten Klassifikationsergebnisse optimiert. Dabei erweist sich die V'envendung folgender Parameter für die Splitbedingungen als günstig:

C1: Verhältnis der mittleren Lecistung von DI im ansteigenden Teil zur mittleren Leistung von $\mathrm{DI}$ in abfallenden Teil der differenzierten peripheren Mulswelle (Verzweigungen 1 und 3)

- 1Q: Impedanzquotient (Verzwcigung 2)

- C2: Verhältnis der mittlercn L_cistung: von 1$) 1 \mathrm{mI}$ ansteigenden Teil der peripheren Pulswalle rut mittk-

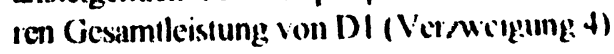


Die lindknoten reprisentieren dic verschiedenen Diagnoscegruppen .,ok", .,palVk“, jentral“ und ,allgemein“. Nach 1()-ficher Kreuzvalidierung werden Fehlklassifikationen gemäl3 Tabcolle 2 notiert, wobei die Spalten dic tatsäichlichen Diagnosegruppen und die Zeilen die Klassilikationsvorschliage des Batumes aus Abbildung 7 repräsentieren. Dic Tabelle ist derart zu lesen, dass beispiclsweise neun gesunde Personen fïlschlicherweise der Klasse ,Zentral" und sichen gesunde Personen der Klasse ,paVk" zugeordnet werden.

Tabelle 2: Fehlklassifikationen nach Kreuzvalidierung

\begin{tabular}{c|cccc}
\hline Fchlklass. in & ok & zentral & paVk & allg. \\
\hline ok & - & 5 & 3 & 3 \\
zentral & 9 & - & 0 & 2 \\
paVk & 7 & 2 & - & 3 \\
allg. & 3 & 3 & 0 & - \\
\hline
\end{tabular}

Die zusätzliche Nutzung zeitaufgelöster Frequenzparameter der zentralen Kurven vermag keine Verbesserung der Klassifikation zu bewirken.

\section{Diskussion}

Durch die Einführung der Wavelet-Transformation konnte die paVk-Diagnostik verbessert werden. Die zeitaufgelösten Frequenzaussagen der Wavelet-Transformation sind ein neuer und Erfolg versprechender Ansatz zur Diagnostik peripherer arterieller Verschlusskrankheiten.

Die Anzahl der fehlklassifizierten Fälle ist für einen klinischen Einsatz der Methodik jedoch noch zu hoch, dafür werden in erster Linie folgende Ursachen gesehen:

Um einen möglichst repräsentativen Stichprobenumfang zu erhalten, wurden auch ältere Probanden in die Untersuchungen mit einbezogen. Die Annahme, dass Probanden a priori, ,gefäßgesund“ sind, kann nicht uneingeschränkt aufrechterhalten werden, da diesbezügliche Untersuchungen nicht explizit durchgeführt wurden. Zukünftige Studien werden diesem Problem Rechnung tragen müssen.

- Aufgrund der kleinen Lernstichprobe, besonders in den Klassen ,zentral", ,paVk" und ,allgemein“, können keine statistisch abgesicherten Aussagen getroffen werden. Die Tendenzen werden allerdings offenkundig. Zur Erhöhung der statistischen Signifikanz ist eine Vergrößerung der Lernstichprobe zukünftig geboten.

- Die Diagnostik der Patienten erfolgte im Rahmen ihrer Therapie nur bei auftretenden Beschwerden. Darüber hinausgehende diagnostische Maßnahmen waren aus Kostengründen leider nicht möglich.

- Die Wirkung der Therapiemaßnahmen und der Einfluss verabreichter Medikamente (ASS, Blutdruckmedikamente und andere) auf die Hämodynamik ist nicht exakt quantifizierbar.

Die Ergebnisse der Kreuzvalidierung sind unter Beachtung der aufgeführten Fehlklassifikationsursachen vielverspre- chend, so dass der extrahierte Merkmalssatz grundsätzlich dic Klassifikation zu leisten im Stande ist. Die vorgestellte Methodik liefert trotz der vorhandenen Fehlklassifikationen im Vergleich zum Stand der Technik deutlich bessere diagnostische Informationen, so dass weitere umfangreiche Studien unter Einbezichung von Referenzuntersuchungen ihre Berechtigung haben.

Entgegen unserer Erwartung lieferten Zeit- und Bildbereichsparameter zentraler Signale keinen Beitrag zur Diagnostik peripherer arterieller Verschlusskrankheiten. Dies ist vermutlich der Tatsache geschuldet, dass 14 der 21 Patienten mit derartigen Krankheitsbildern vasodilatorisch wirkende Medikamente (Nitrate) erhalten.

Andere, im Vergleich zu Abbildung 7, berechnete Klassifikationsbäume mit vergleichbaren Klassifikationsergebnissen verwenden mitunter andere Merkmale zur Entscheidungsfindung. Bei vielen Bäumen wurde AQ als Splitbedingung der ersten Verzweigung verwendet. Hieraus kann geschlossen werden, dass bei Verwendung der vorgestellten Merkmale der anakrote Schenkel der peripheren Kurven diagnostisch relevanter ist als der katakrote. Weiterhin bleibt festzuhalten, dass zeitaufgelöste Frequenzaussagen über den Anstieg der peripheren Signale bei Verwendung von Klassifikationsbäumen diagnostisch signifikanter sind als Anstiegsparameter des Zeitbereichs.

Um den Informationsunterschied des katakroten Schenkels bei Patienten mit unterschiedlichen Gefäßerkrankungen (s. Abbildung 3) besser nutzbar zu machen, werden bei zukünftigen Untersuchungen vorhandene Merkmale weiterentwickelt resp. neu generiert werden müssen. Vorstellbar ist die Verwendung von Wavelet-Packets oder anderen Verfahren der Zeit-Frequenzanalyse. Die Anwendung derartiger Methoden lässt eine Verbesserung der Klassifikationsergebnisse erwarten.

\section{Literaturverzeichnis}

[1] Statistisches Bundesamt, Gesundheitsbericht für Deutschland, ch. 5, pp. 27-42, 1998

[2] J. Ranft, "Häufigkeit und Prognose arterieller Durchblutungsstörungen", http://www.angiologie-online.de/AVK.htm, 1.5.2000

[3] J. Ranft, "Arterielle Verschlusskrankheiten", http://wurw.angiologie-online.de/Atherosklerose.htm, 9.10.2000

[4] R. Schüler, Apparative Gefäßdiagnostik: Untersuchungsmethoden und deren Anwendung, Ilmenau: ISLE Verlag, 1998. 